\title{
Dynamic model of the left ventricle for use in simulation of myocardial perfusion SPECT and gated SPECT
}

\author{
S. Bullich \\ Unitat de Biofísica i Bienginyeria, Facultat de Medicina, Universitat de Barcelona, Casanova 143, 08036 \\ Barcelona, Spain \\ D. Ros \\ Unitat de Biofísica i Bienginyeria, Facultat de Medicina, Universitat de Barcelona, \\ and Institut d'Investigacions Biomèdiques August Pi i Sunyer (IDIBAPS), Casanova 143, 08036 \\ Barcelona, Spain
}

A. Cot

Departament de Física i Enginyeria Nuclear, Universitat Politècnica de Catalunya, Diagonal 647, 08028 Barcelona, Spain

C. Falcón

Unitat de Biofísica i Bienginyeria, Facultat de Medicina, Universitat de Barcelona, Casanova 143, 08036

Barcelona, Spain

A. Muxí

Servei de Medicina Nuclear, Hospital Clínic i Provincial de Barcelona, Villaroel 170; 08036 Barcelona, Spain

J. Paviaa)

Servei de Medicina Nuclear, Hospital Clínic i Provincial de Barcelona, and Institut d'Investigacions

Biomèdiques August Pi i Sunyer (IDIBAPS), Villarroel 170, 08036 Barcelona, Spain

(Received 23 July 2002; accepted for publication 13 May 2003; published 9 July 2003)

Simulation is a useful tool in cardiac SPECT to assess quantification algorithms. However, simple equation-based models are limited in their ability to simulate realistic heart motion and perfusion. We present a numerical dynamic model of the left ventricle, which allows us to simulate normal and anomalous cardiac cycles, as well as perfusion defects. Bicubic splines were fitted to a number of control points to represent endocardial and epicardial surfaces of the left ventricle. A transformation from each point on the surface to a template of activity was made to represent the myocardial perfusion. Geometry-based and patient-based simulations were performed to illustrate this model. Geometry-based simulations modeled (1) a normal patient, (2) a well-perfused patient with abnormal regional function, (3) an ischaemic patient with abnormal regional function, and (4) a patient study including tracer kinetics. Patient-based simulation consisted of a left ventricle including a realistic shape and motion obtained from a magnetic resonance study. We conclude that this model has the potential to study the influence of several physical parameters and the left ventricle contraction in myocardial perfusion SPECT and gated-SPECT studies. (C) 2003 American Association of Physicists in Medicine. [DOI: 10.1118/1.1589497]

Key words: left ventricle, model, Monte Carlo, SPECT, GSPECT

\section{INTRODUCTION}

Simulation is a useful tool for elucidating the effect of radiation-matter interactions on images and assessing the accuracy of quantification algorithms. Although anthropomorphic phantoms constituted an early approach to the simulation of cardiac SPECT studies, they were static models that did not permit a simulation of the cardiac cycle. Some attempts to simulate movements in anthropomorphic phantoms have led to simple models of the cardiac cycle. ${ }^{1,2}$ However, these models failed to accurately simulate realistic movements such as rotation, change of position due to respirationinduced movement, or anomalous movements caused by ischaemia. Moreover, these models are adapted to a particular anatomy and physiology and are not sufficiently versatile to simulate changes in shape and perfusion.

Numerical simulation is another possibility. In essence, numerical simulation consists of mathematically representing two physical distributions: the geometrical characteristics of the radiation source (activity map) and the spatial distribution of attenuating material (attenuation map). Information included in activity and attenuation maps is used by deterministic or Monte Carlo projectors during the image formation process. Existing models fall into two groups: geometrybased and voxel-based. Geometry-based phantoms ${ }^{3-5}$ are derived from simple equations. The main advantage of this type is that different anatomical variations and images can be generated at multiple resolutions. Voxel-based phantoms ${ }^{6,7}$ are generally extrapolated from patient data, and are fixed to a particular anatomy and resolution. It is possible to make geometry-based phantoms reasonably realistic but they will not be as realistic as voxel-based phantoms. Recently some attempts at building hybrid phantoms that preserve the best characteristics of voxel-based and geometry-based models have aroused considerable interest. ${ }^{8,9}$ Both kinds of phan- 
toms have been used to analyze the effects of anatomical variations in cardiac SPECT images ${ }^{10}$ and gated SPECT ${ }^{11,12}$ images.

Despite the large number of recent improvements, some features of cardiac SPECT and gated-SPECT studies remain nonrealistically simulated. Even though clinical results have clearly demonstrated that ischaemia causes immediate and severe dysfunctional contraction, ${ }^{13,14}$ simultaneous simulation of ischaemia and anomalous movements remain underexplored. This work is focused on reducing the limitations in two areas. The first area is the creation of a model of the left ventricle (LV), which allows the simulation of left ventricular motion in diseased states, taking care to simulate not only the movement but also the perfusion. The second area is that, when a stable tracer is being simulated, the total and the regional activity within the LV should remain constant throughout the cardiac cycle.

\section{METHODS}

\section{A. Characteristics of the model}

In order to model a dynamic LV, a number of characteristics should be taken into account. In this section we seek to provide a summary of the main characteristics of the numerical phantom.

(1) Anatomy. We assume that in myocardial perfusion SPECT and gated-SPECT studies, only the left ventricle is clearly observable, and only this will be relevant in the simulation. This approximation has also been used in earlier papers. ${ }^{15-17}$ Such a simplification will be acceptable in most perfusion and gated-SPECT studies and allows us to concentrate on improving the left ventricle modeling. This approximation will not be acceptable in cases where high activity in the right ventricle can influence LV activity. For example, after injection of the tracer, a right ventricle blood pool can affect images of the LV.

(2) Left ventricle contraction. LV movement consists of the contraction of the cardiac walls. During this contraction the apex remains almost static, whereas the base moves toward the apex, forcing blood into the circulatory system. As a result of contraction, the myocardial wall increases its thickness, with a significant decrease in the perimeter of the endocardial wall and a smaller decrease in the epicardial wall perimeter. In addition, other movements such as rotation or movements due to respiration could be included. An indepth study of the left ventricle movement can be found in Refs. 18-21.

(3) Malfunctional contraction. From a kinetic point of view, a diseased state of the left ventricle results in anomalous movements. ${ }^{13,14}$ Some interesting cases to simulate are akinesis, hypokinesis, and diskinesis and their associated changes in thickening.

(4) Rotational movement. It has been established that during systole the base of the LV moves clockwise, and the apical region moves anticlockwise. These movements of rotation invert during diastole and have been quantified, in normal patients, between $0^{\circ}$ and $7^{\circ} \cdot{ }^{22-25}$ The midventricular region remains almost stationary during systole.

(5) Respiratory movement. Breathing affects the motion of the heart. The diaphragm moves up and down between $1-10 \mathrm{~cm}$ during breathing ${ }^{9,26}$ and the heart moves in a similar fashion. Respiratory motion can alter left ventricular function and cause artifacts in myocardial SPECT, leading to a misinterpretation of images, especially in the area of the inferior wall of the LV. ${ }^{27}$

(6) Activity. A fundamental condition to be modeled-when a stable tracer is being simulated-is that the total and regional activities of the heart during the cardiac cycle should remain constant. This means that, although the LV moves and thickens, the amount of activity in a region of the LV does not change during the cardiac cycle.

\section{B. Input parameters}

To generate the phantom a number of parameters must be supplied to the algorithms.

(1) Parameters representing endo- and epicardial surfaces. In this model, the endocardial and epicardial surfaces of the LV are described using a number of control points (CP). Each CP is specified using four coordinates: three for space and one for time $(r, \theta, \varphi, t)$. CP must be carefully placed since regions of the surface without $\mathrm{CP}$ will not be satisfactorily modeled. The choice of CPs could be obtained from equations representing the surfaces, from images with good anatomical resolution such as magnetic resonance (MR) or be specified by the operator. Figure 1 shows two examples of different ways of defining the coordinates of CPs (both are used in the following section "Illustrative models"). The upper half of the image shows two short-axis slices of a MR image of a heart at end-systole and end-diastole. CPs used to represent endo- and epicardial surfaces are presented. Different short-axis slices from base to apex should be used to determine the three-dimensional coordinates of the CPs. The lower half of the image shows a geometrical model to represent endo and epicardial surfaces. CPs used to represent endo and epicardial surfaces are distributed uniformly over the surfaces.

(2) Parameters representing perfusion. Perfusion is represented in a two-dimensional template. As the ventricular wall moves and thickens, the CPs move in space, but the activity remains fixed to the myocardial wall. It is necessary for each $\mathrm{CP}$, regardless of its position during the cardiac cycle, to transform into the same point on the template. A mathematical transformation between $\mathrm{CP}$ and points on the template is used to determine the amount of activity in the myocardium. This transformation is found by using as input parameters pairs of coordinates on the surface $(\theta, \varphi, t)$ and their corresponding coordinates $(x, y)$ on the template. The mathematics used to calculate this transformation is detailed in the Appendix. Note that this transformation does not need to be bijective and some points can be transformed to more than one point on the template, as occurs at the apex. 

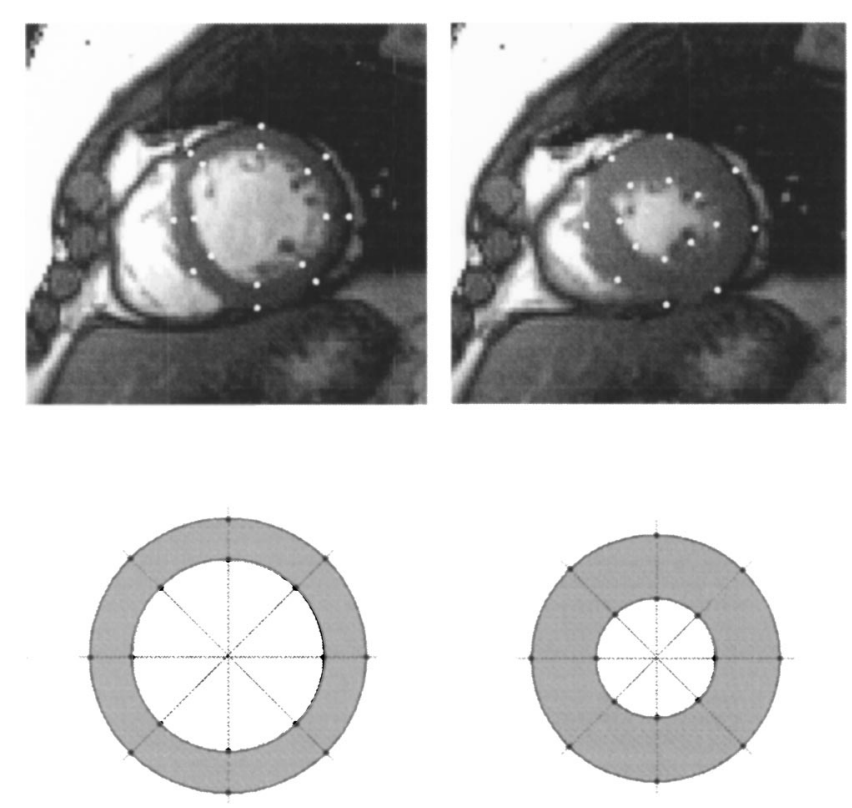

FIG. 1. An example of the methods used to define the coordinates of CPs. The upper half of the image shows two short-axis slices of a MR image of a heart at the end systole and end diastole. The lower half of the image shows a geometrical model to represent endo- and epicardial surfaces. CPs are distributed uniformly over the surfaces. Different short-axis slices from the base to the apex should be used to determine the three-dimensional coordinates of the CPs.

This will not produce any problems if care is taken not to introduce incongruencies in the template. To make the model easier to compute, we consider the transformed points on the template ordered in the vertex of a rectangular array. This restriction helps to simplify the implemented algorithms but does not introduce limitations to the shape and contraction of the modeled LV.

Figure 2 shows a graphical representation of the transformation using a geometrical model. A LV with a number of CPs on the surface is shown on the left. A template of activity and the transformed $\mathrm{CP}$ are presented on the right. The template of activity can be fixed by the operator or obtained
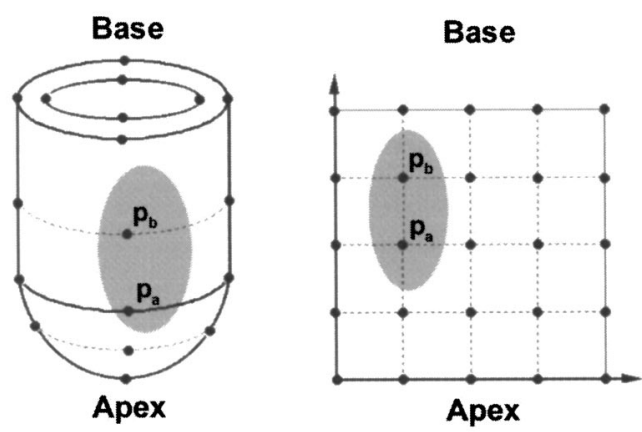

FIG. 2. An illustration showing the distribution of the CPs and a template of activity. On the left, a LV with a number of control points on the surface is displayed. On the right, these control points are transformed onto the template. Note that the activity is represented on the template and transformed onto the LV. from patient data. In this paper, the template was represented by a $32 \times 32$ matrix and stored in a text file. The matrix was generated automatically to represent uniform perfusion. Ischaemia was simulated by manual modification of some elements of the matrix.

\section{Activity and attenuation maps}

The numerical phantom of the LV consists of threedimensional matrices representing activity and attenuation distributions. Activity and attenuation maps are needed for each moment in the cardiac cycle to simulate a gated-SPECT study. Three steps are followed to fix the numerical activity to each voxel: (1) The equations describing the endocardial and epicardial surfaces are determined for each gate. These equations were obtained from the $\mathrm{CP}$ and the transformation by using bicubic splines: ${ }^{28}$

$$
\begin{aligned}
& r_{\mathrm{en}}^{t}=r_{\mathrm{en}}^{t}(\theta, \varphi)=r_{\mathrm{en}}^{t}\left(\theta_{\mathrm{en}}(x, y), \varphi_{\mathrm{en}}(x, y)\right), \\
& r_{\mathrm{ep}}^{t}=r_{\mathrm{ep}}^{t}(\theta, \varphi)=r_{\mathrm{ep}}^{t}\left(\theta_{\mathrm{ep}}(x, y), \varphi_{\mathrm{ep}}(x, y)\right),
\end{aligned}
$$

where $r_{\mathrm{en}}^{t}$ and $r_{\mathrm{ep}}^{t}$ are the equations of the endocardial and epicardial surfaces at time $t$. (2) For each voxel of coordinates $(r, \theta, \varphi, t)$, we verify whether or not it falls within the LV myocardium $\left(r_{\mathrm{en}}^{t}(\theta, \varphi)<r<r_{\mathrm{ep}}^{t}(\theta, \varphi)\right)$. The coordinates of all these voxels in the myocardium wall are transformed to the template, as described in the Appendix. The activity for these voxels is obtained from the template. (3) Finally, the activity map is normalized to the regional activity of the LV. To this end, the template is divided into sections. Each section corresponds to a part of the LV and can be normalized individually.

Thorax information should be included in order to obtain the total activity map. The mathematical cardiac torso phantom $(\mathrm{MCAT})^{5}$ was employed in this work. Thus, the total activity was obtained by inserting our LV inside the MCAT phantom in the correct position and orientation. The relative activity per voxel assigned to the lung and the rest of the body (including ventricular chamber) was $15 \%$ of the normal myocardial wall. The liver and kidney were not included.

The attenuation map used is the one provided by MCAT. Attenuation coefficients for soft tissue, lung, and spine were $0.150,0.044$, and $0.189 \mathrm{~cm}^{-1}$.

To generate a nongated study, all the activity maps can be averaged together during simulation. To generate a gated study, individual time samples are used separately or small groups of adjacent time samples are averaged to simulate movement in every time interval.

\section{Projections and reconstruction}

Simulation of projections was performed with Monte Carlo methods by using the SimseT code. ${ }^{29,30}$ Photons of 140 $\mathrm{keV}$ were used to simulate a Technetium $\left({ }^{99 \mathrm{~m}} \mathrm{Tc}\right)$ acquisition. Thallium $\left({ }^{201} \mathrm{Tl}\right)$ decays by electron capture, emitting x-ray and gamma photons, with $95 \%$ of the emission between 69 and $82 \mathrm{keV}$. For the sake of simplicity, photons of $71 \mathrm{keV}$ were simulated in a thallium acquisition. In both cases, a low-energy high-resolution collimator and an energy window 


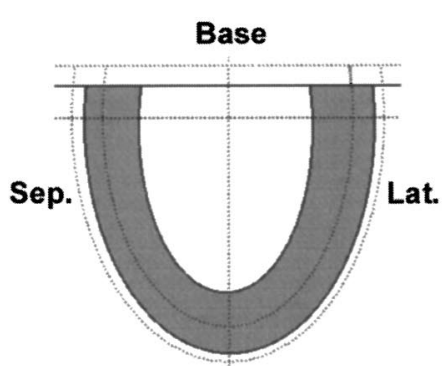

Apex
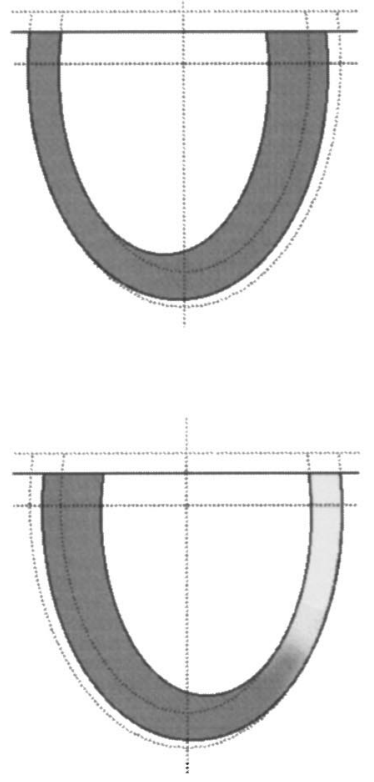

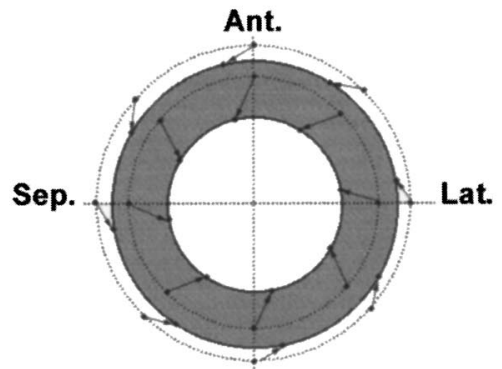

Inf.
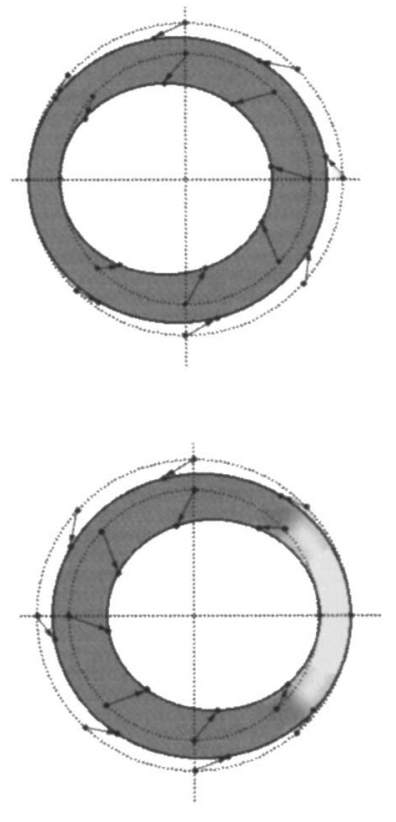

FIG. 3. The horizontal long axis (HLA), vertical long axis (VLA), and mid-ventricular short axis (SA) diagrams of a geometric left ventricle. The continuous line shows the end systole and the dotted line shows the end diastole. The upper row of the figure (simulation A) represents normal contraction. The motion includes a small movement of the epicardial surface and a larger movement of the endocardial surface. The apex remains almost static and the base moves toward the apex. Rotational movement of the control points was included and is represented by arrows. The central row of the figure (simulation B) shows a patient with a reduced regional function in the septum. The lower row of the figure (simulation C) represents a patient with ischaemia and reduced regional function in the lateral wall (lateral akinesis). The light gray region represents the placement of the ischaemia. of $20 \%$ were employed. The projections were simulated on a $64 \times 64$ matrix with a pixel size of $0.634 \mathrm{~cm}$ to simulate standard SPECT and gated-SPECT formats. The postprocessing of all the simulations was performed by using a filtered-backprojection algorithm with a Butterworth filter (order: 3.5 , cut-off frequency: $0.44 \mathrm{~cm}^{-1}$ ).

\section{E. Illustrative models}

To illustrate this model, five studies were simulated. These were created with 48 CPs distributed on the surface. A $128 \times 128 \times 128$ matrix with a voxel size of $0.31 \mathrm{~cm}$ was employed for activity and attenuation maps. The main characteristics of each simulation were the following.

(a) Case A. Well-perfused patient with normal regional function. In this case, a geometric simulation of the LV of a patient with normal perfusion and uniform wall thickening and contraction was performed. The geometry of this LV was fixed by the operator and consisted of two ellipsoids truncated by the valvular plane. The data used to create the geometric model (size, radial, and longitudinal contraction, thickness,...) was fixed to be similar to those of a real left ventricle. The contraction, thickness, and ejection fraction can be calculated analytically from the equation of the truncated ellipsoids used to generate the CPs. Figure 3 shows a scheme of the LV in which CPs and their motion is represented. The cardiac cycle was divided into eight intervals where the end diastole was the first moment of the cycle and the end systole was the third moment of the cycle. The contraction was simulated by a small movement of the epicardial surface and a larger movement of the endocardial surface. The apex remained almost static and the base moved toward the apex. The pattern of movement modeled a quick contraction from end diastole to end systole and a slow relaxation. Rotational motion was simulated by adding a rotational movement to the CPs. During systole, the CPs of the base of the LV moved clockwise, and the CPs of the apical region moved anticlockwise. These rotational movements inverted during diastole. The maximum rotation was $7^{\circ}$. Respiratory-induced motion was not included. Each interval of the cardiac cycle was averaged to form the mean activity to simulate a myocardial perfusion SPECT acquisition. The continuous line in Fig. 4 shows the LV volume during the cardiac cycle. The volume curve and ejection fraction was calculated analytically from the equation of the truncated ellipsoid 


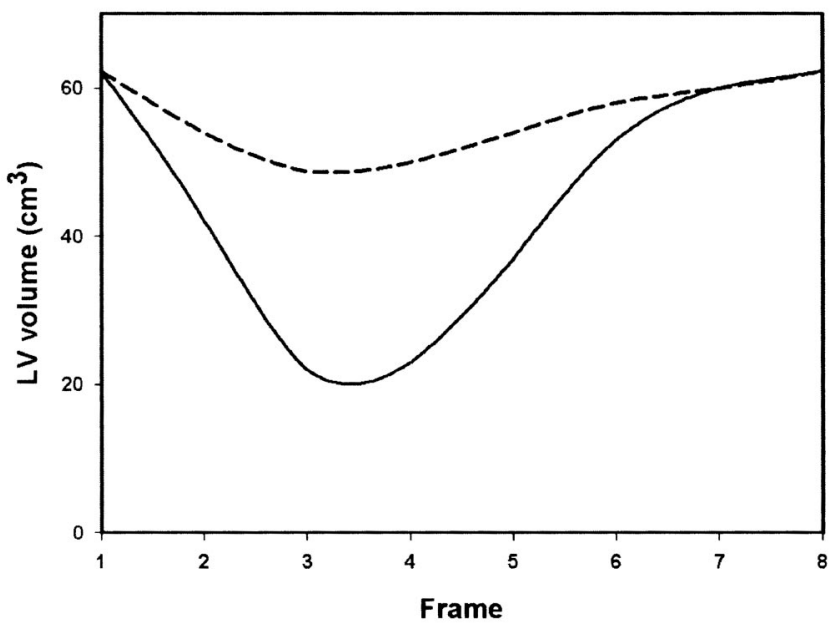

FIG. 4. The figure representing the simulated LV volume during the cardiac cycle for a patient with a normal contraction (continuous line) and with abnormal contraction (dashed line).

used to generate the CPs. The ejection fraction was $65 \%$. CPs were distributed uniformly on the surfaces and activity template represented a uniform activity. The subdivision of the template was performed in 30 square-shaped areas.

(b) Case B. Well-perfused patient with reduced regional function. This case simulated a patient with normal perfusion but without wall thickening and contraction in the septum. The geometry and perfusion of this LV was the same as in case A. CPs representing the lateral wall remained static between the end systole and end diastole, thus simulating a region without contraction and thickening. The horizontal long axis and short axis of the end systole and end diastole can be seen in Fig. 3. The dashed line in Fig. 4 shows the LV volume during the cardiac cycle. The ejection fraction was $21 \%$, simulating a diseased patient. CPs were distributed uniformly on the surfaces and the activity template represented a uniform activity. The subdivision of the template was performed in 30 square-shaped areas.

(c) Case C. Ischaemic patient with reduced regional function. This case simulated a patient with abnormal perfusion and without wall thickening or contraction in the lateral wall. The geometry and contraction of this LV was the same as in case B. Ischaemia was simulated by reducing activity by $25 \%$ in the regions of the template representing the lateral wall. A horizontal long axis and short axis at the end systole and end diastole can be seen in Fig. 3. The dashed line in Fig. 4 shows the LV volume during the cardiac cycle. The ejection fraction was $21 \%$.

(d) Case D. Model including tracer kinetics. This case simulates a number of acquisitions of a thallium-201 $\left({ }^{201} \mathrm{Tl}\right)$ SPECT. After intravenous administration, ${ }^{201} \mathrm{Tl}$ is rapidly taken up by myocardial tissue and other systemic organs in proportion to blood flow. After an initial distribution only $2 \%$ of the injected dose of thallium-201 remains in the blood pool. The equilib- rium phase or delayed distribution is reached several hours after intravenous administration. The ${ }^{201} \mathrm{Tl}$ uptake in the delayed redistribution phase is due to the washout of thallium from normally-perfused zones and with delayed accumulation or slow ${ }^{201} \mathrm{Tl}$ washout from the hypoperfused zones. ${ }^{31}{ }^{201} \mathrm{Tl}$ kinetics for different tissues was approximated by the function

$c(t)=c_{\max }\left(\frac{t}{\alpha \beta}\right)^{\alpha} e^{-t / \beta}+c_{0}\left(1-e^{-t / \tau}\right)$,

where $c(t)$ indicates the concentration of ${ }^{201} \mathrm{Tl}$ in the tissue and $c_{\max }, \alpha, \beta, c_{0}, \tau$ are the parameters that can be adjusted to model different ${ }^{201} \mathrm{Tl}$ kinetics. The first part of the equation represents ${ }^{201} \mathrm{Tl}$ uptake and the second part allows us to model washout. To simulate normal tissue, the parameters chosen were $c_{\max }=5$, $\alpha=2, \beta=8, c_{0}=1000, \tau=5$. To simulate a reversible defect, the parameters selected were $c_{\max }=3.6, \alpha=2$, $\beta=8, c_{0}=900, \tau=5$. To simulate an irreversible defect, the parameters chosen were $c_{\max }=0, \alpha=2, \beta=8, c_{0}$ $=400, \tau=10$. Figure 5 illustrates ${ }^{201} \mathrm{Tl}$ kinetics for different degrees of defects (normal tissue, reversible defect and irreversible defect). The time where a SPECT acquisition was performed is marked in gray. Two simulations were performed. The first simulation included a geometrical model, as described in model A (but without contraction) with a reversible defect in the anterior wall. The second simulation modeled an irreversible defect in the anterior wall. In both simulations, the rest of the myocardium had normal tissue. Each SPECT acquisition was obtained by sampling the activity in five-time frames during the acquisition and by averaging them.

(e) Case E. Patient-based simulation. This case simulates a normal LV with information obtained from a real patient. A gated MR image of a normal subject was acquired using a $\mathrm{Tl}$ sequence in eight intervals. The LV was reoriented to obtain the short axis. This simulation included contraction and rotational motion obtained from the MR image by placing the CPs on the surface of the left ventricle. Forty-eight CPs per interval were manually placed on the short axis. However, without specific landmarks on the heart surface, the real contraction and rotational motion were only approximated in the model. Only large geometrical characteristics were considered; papillary muscles were not simulated. The template used to fix the perfusion was uniform.

\section{RESULTS}

The results of the illustrative models are shown in this section. Figure 6 gives the results of cases A, B, and C. This figure shows five slices of the simulated LV (three short axis, horizontal axes, and vertical axes). The first row (case A) shows the results of the simulation of a patient with a wellperfused myocardium. A decrease in perfusion can be seen from apex to base due to attenuation. The second row (case 


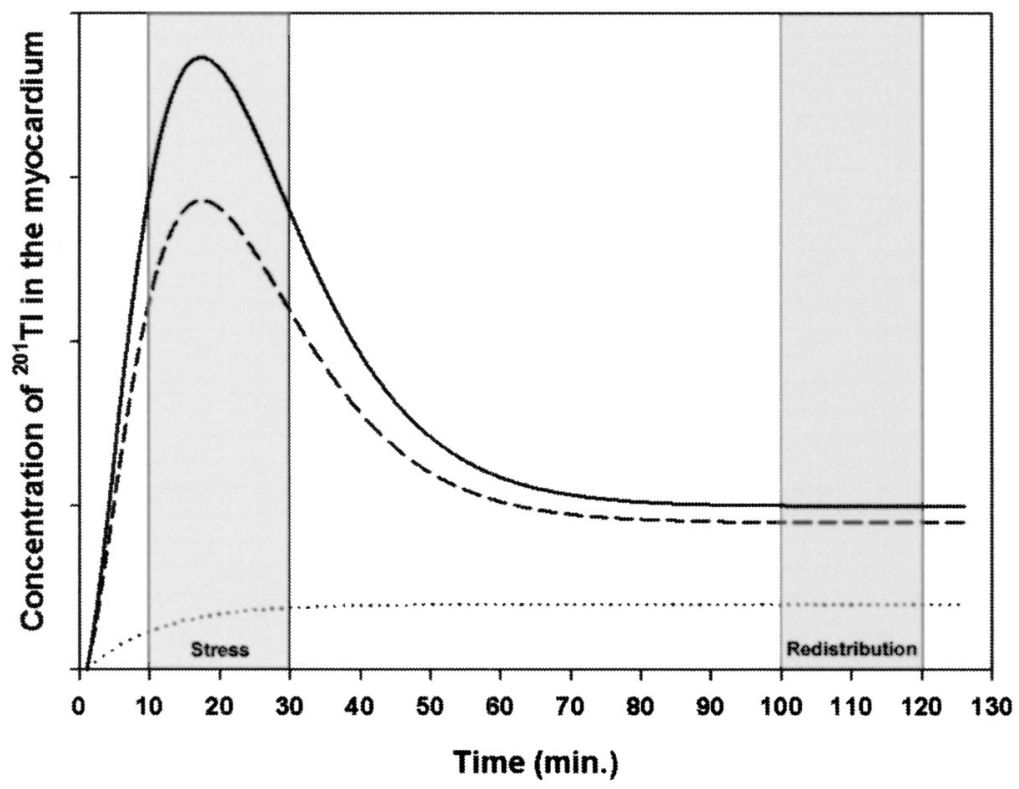

FIG. 5. A figure representing the simulated thallium-201 kinetics in the myocardium. A continuous line represents tracer kinetics for a normal tissue, a dashed line represents tracer kinetics for a tissue affected by a reversible defect, and a dotted line represents tracer kinetics for a tissue affected by a persistent defect. The time intervals where the SPECT acquisitions were performed are marked in gray.

B) shows the results of the simulation of a patient with normal perfusion but reduced regional function in the septum. Despite simulating the activity of case A, a lower intensity is observed in the septum. This can be attributed to the fact that the partial volume effect has a higher impact on the septum than on the lateral wall. Thus, example B clearly shows that changes in movement or contraction patterns can yield mis-

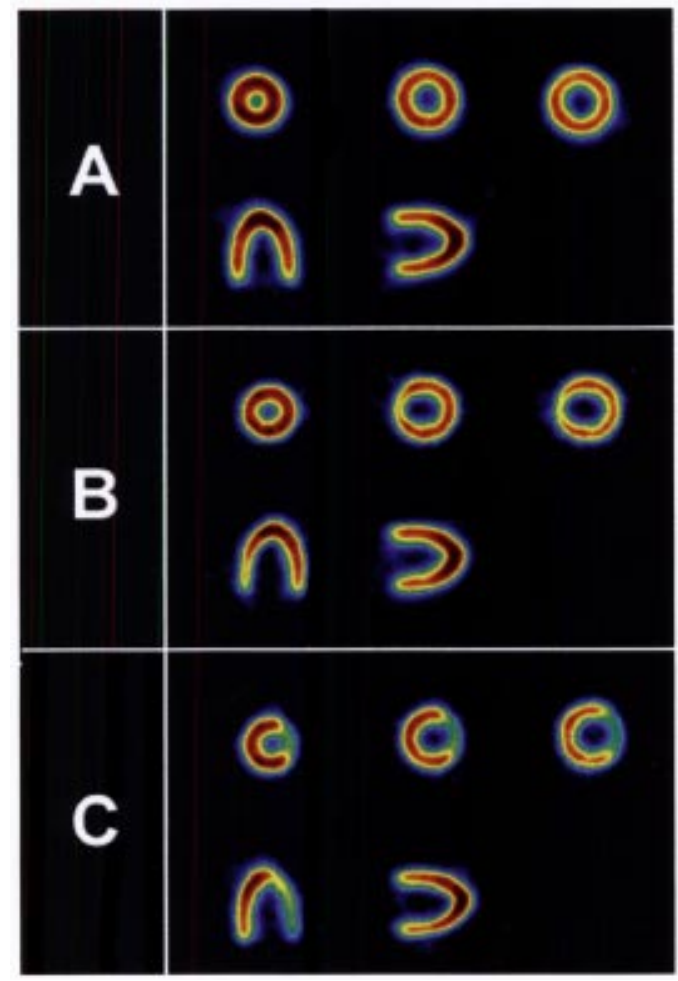

FIG. 6. Horizontal long axis (HLA), vertical long axis (VLA), and short axis (SA) images of the simulated phantoms. The upper row shows a normal simulation (case A), the middle row shows a normal simulation with reduced regional function (case B), and the lower row shows an ischaemic simulation with reduced regional function (case $\mathrm{C}$ ). leading perfusion levels. Although this error would probably not be significant enough to result in a wrong diagnosis, it might influence the calculation of perfusion changes between two studies of the same patient.

The third row (case $\mathrm{C}$ ) shows the simulation of a patient with ischaemia in the lateral wall and reduced regional function. This simulates a case that is often encountered in clinical practice. Further studies including varying degrees of hypokinesis and ischaemias in various regions should be undertaken in order to draw conclusions.

Figure 7 gives the results of simulation D. This figure shows a horizontal long axis, a vertical long axis and three

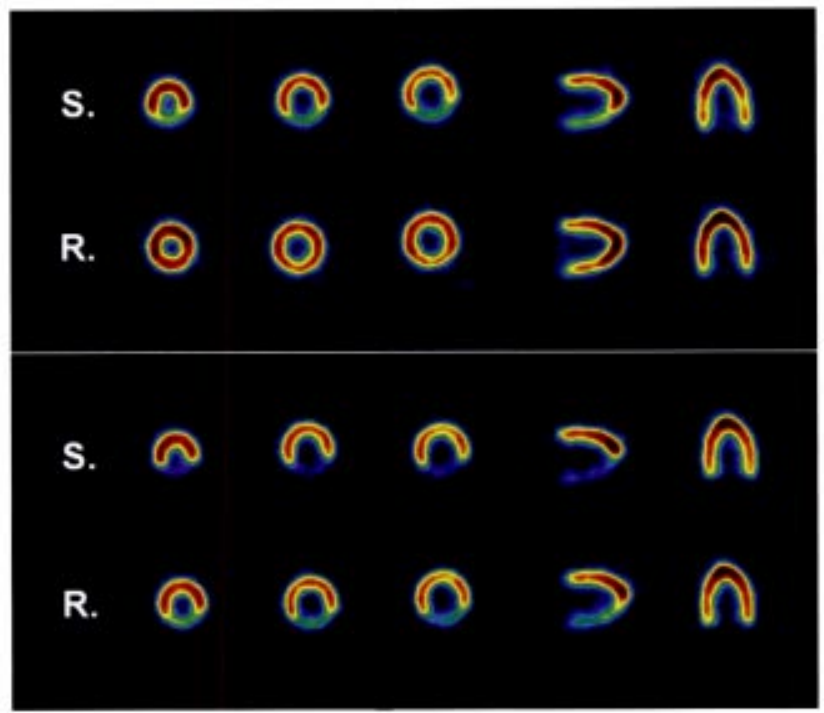

FIG. 7. A figure representing a SPECT acquisition, including the tracer kinetics. The LV is displayed in stress $(\mathrm{S})$ and in redistribution (R). The upper half of the figure shows a LV with a reversible defect in the anterior wall and normal tissue in the rest of the myocardium. The lower half of the figure shows a LV with an irreversible defect in the anterior wall and normal tissue in the rest of the myocardium. 


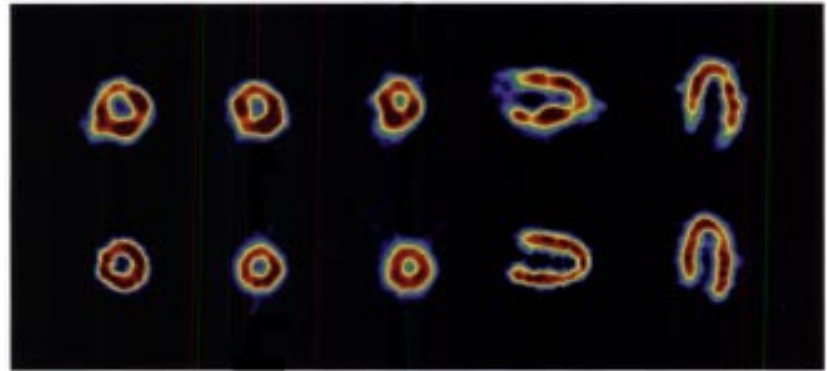

FIG. 8. A figure showing the results of simulation E. The upper row displays a myocardial perfusion SPECT of a patient and the lower row displays the simulated LV with the CPs obtained from a MR image of the same patient.

short axes of the LV for each simulation. The upper half presents a LV with a reversible defect in the anterior wall and normal tissue in the rest of the myocardium. This left ventricle is represented in stress $(\mathrm{S})$ and in redistribution $(\mathrm{R})$ situations. The normal tissue shows a uniform uptake and also a uniform redistribution in all the walls of the LV. The reversible defect in the anterior wall displayed a reduced uptake in this wall in comparison with the normal region. In the redistribution, both regions are indistinguishable. The lower half of the figure shows a LV with an irreversible defect in the anterior wall and normal tissue in the rest of the myocardium. The irreversible defect displayed a lack of uptake and no redistribution.

Figure 8 gives the result of the simulation of case E obtaining the CPs from a gated MR image. The upper row shows the myocardial perfusion SPECT of the patient and the lower row displays the simulated LV. Large anatomical variations can be taken into account to generate a realistic model. This is the case of thinning of the myocardial wall and shortening of the septum.

\section{DISCUSSION}

A dynamic LV model was designed and implemented and some examples are reported. Our initial results suggest that the phantom has the potential to simulate normal LV as well as LV affected by different cardiac disorders such as those affecting contraction, morphology, and tracer kinetics.

Geometry-based simulations modeled normal and pathological LVs with contraction and tracer kinetics disorders. In this paper, these disorders were simulated separately in different models. However, the methodology used to generate the LV does not exclude the possibility of including contraction, hypoperfusion, and tracer kinetics in the same model.

Respiratory-induced movement was not included. Nevertheless, it has been treated by other authors. ${ }^{26}$ Their methodology has the potential to be included in our model so as to simulate respiratory-induced movement.

A patient-based simulation was generated using anatomical information obtained from gated MR images. Figure 8 shows that this methodology has the potential to create a realistic model for the LV including the contraction and change in shape. Anatomical variations can be modeled using several sets of patient data. However, the lack of some information limits the realism of the simulation. Without specific landmarks on the heart surface, no realistic time correspondence can be established for the CPs from frame to frame. In order to realistically and accurately model the motion of a CP from frame to frame, tagged MRI data would have to be used. In addition, a uniform perfusion was simulated which is not the case in actual patient studies. Other sources of discrepancy between modeled and real images are that abdominal structures and papillary muscles were not considered.

Applications of this phantom could include the evaluation of (1) alterations of the apparent distribution of activity due to contraction of the heart; (2) the accuracy of methods for determining cardiac global and regional function and (3) four-dimensional reconstruction methods of gated cardiac SPECT. Moreover, PET and dynamic PET studies could be simulated using PET projectors.

\section{ACKNOWLEDGMENTS}

This work was supported in part by Ministerio de Ciencia y Tecnologia, MCYT (SAF99-0137 and SAF2002-04270C02-01/02) and Fondo de Investigación Sanitaria, FIS (G03/ 185). The work of S. Bullich was supported by a grant from the Institut d'Investigacions Biomèdiques August Pi i Sunyer (IDIBAPS).

\section{APPENDIX: TRANSFORMATION BETWEEN 3-D SPACE AND 2-D TEMPLATE}

When the ventricular wall moves and thickens, the activity remains fixed to the myocardial wall. It is necessary to carry out a transformation between points on the twodimensional template and $\mathrm{CP}$. This transformation requires a $\mathrm{CP}$ to transform to the same point on the template regardless of its movement. This transformation can be represented mathematically as follows:

$$
\begin{gathered}
\tau_{\mathrm{en}}^{t}:\left(x_{i}, y_{i}\right) \rightarrow\left(\theta_{\mathrm{en}, i}^{t}, \varphi_{\mathrm{en}, i}^{t}\right) \\
\tau_{\mathrm{ep}}^{t}:\left(x_{i}, y_{i}\right) \rightarrow\left(\theta_{\mathrm{ep}, i}^{t}, \varphi_{\mathrm{ep}, i}^{t}\right),
\end{gathered}
$$

where $\left(x_{i}, y_{i}\right)$ are points on the template, $\left(r_{\mathrm{en}}, \theta_{\mathrm{en}}, \varphi_{\mathrm{en}}, t\right)$ are $\mathrm{CP}$ on the endocardial surface, and $\left(r_{\mathrm{ep}}, \theta_{\mathrm{ep}}, \varphi_{\mathrm{ep}}, t\right)$ are points on the epicardial surface as represented in Fig. 1. With $x$ and $y$ as the ordinates in the vertexes of a rectangular array, several bicubic splines ${ }^{28}$ can be adjusted to determine the transformation for the endocardial surface $\left(\theta_{\mathrm{en}, i}^{t}\right.$ $\left.=\theta_{\mathrm{en}, i}^{t}\left(x_{i}, y_{i}\right), \varphi_{\mathrm{en}, i}^{t}=\varphi_{\mathrm{en}, i}^{t}\left(x_{i}, y_{i}\right)\right)$ and epicardial surface $\left(\theta_{\mathrm{ep}, i}^{t}=\theta_{\mathrm{ep}, i}^{t}\left(x_{i}, y_{i}\right), \varphi_{\mathrm{ep}, i}^{t}=\varphi_{\mathrm{ep}, i}^{t}\left(x_{i}, y_{i}\right)\right)$.

The inverse transformation to find $(x, y)$ given $(\theta, \varphi)$ can be obtained by minimizing the following two-dimensional functions:

$$
\begin{aligned}
& g_{\mathrm{en}, \theta, \varphi}^{t}(x, y)=\left[\left(\theta-\theta_{\mathrm{en}, i}^{t}(x, y)\right)^{2}+\left(\varphi-\varphi_{\mathrm{en}, i}^{t}(x, y)\right)^{2}\right]^{1 / 2}, \\
& g_{\mathrm{ep}, \theta, \varphi}^{t}(x, y)=\left[\left(\theta-\theta_{\mathrm{ep}, i}^{t}(x, y)\right)^{2}+\left(\varphi-\varphi_{\mathrm{ep}, i}^{t}(x, y)\right)^{2}\right]^{1 / 2} .
\end{aligned}
$$

This minimization has been implemented with the Simplex algorithm. ${ }^{28}$

Using this methodology, two transformations can be found: a transformation from the points of the endocardial 

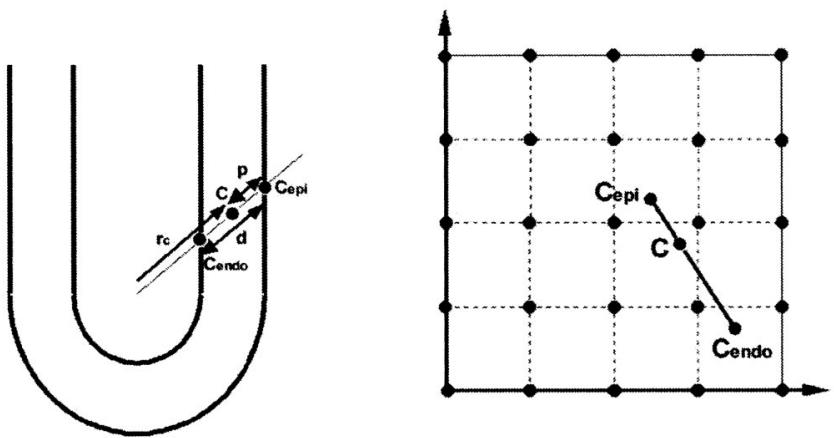

FIG. 9. A graph showing the transformation of a point within the ventricle to the template.

surface to the template and a transformation from the points of the epicardial surface to the template. The transformation from a point $(c)$ with coordinates $\left(c_{r}, c_{\theta}, c_{\varphi}\right)$ between the endo- and epicardial surfaces was implemented as the weighted average of the transformation of this point by both transformations. The mathematical expressions are

$$
c^{\prime}=(d-p) / d \cdot \tau_{\mathrm{en}}^{t}(c)+(p / d) \cdot \tau_{\mathrm{ep}}^{t}(c),
$$

where

$$
\begin{aligned}
& d=r_{\mathrm{ep}}^{t}(c)-r_{\mathrm{en}}^{t}(c), \\
& p=r_{\mathrm{ep}}^{t}(c)-r_{c},
\end{aligned}
$$

and using $r$ as defined in the article:

$$
\begin{aligned}
& r_{\mathrm{en}}^{t}=r_{\mathrm{en}}^{t}(\theta, \varphi)=r_{\mathrm{en}}^{t}\left(\theta_{\mathrm{en}}(x, y), \varphi_{\mathrm{en}}(x, y)\right), \\
& r_{\mathrm{ep}}^{t}=r_{\mathrm{ep}}^{t}(\theta, \varphi)=r_{\mathrm{ep}}^{t}\left(\theta_{\mathrm{ep}}(x, y), \varphi_{\mathrm{ep}}(x, y)\right) .
\end{aligned}
$$

A graphical representation of the transformation of a point within the ventricle to the template can be found in Fig. 9.

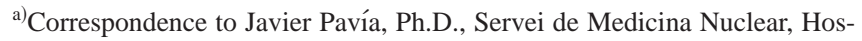
pital Clínic i Provincial de Barcelona, Villarroel 170, 08036 Barcelona, Spain. Electronic mail: pavia@medicina.ub.es

${ }^{1} \mathrm{~N}$. Kubo et al., "A new dynamic myocardial phantom for the assessment of left ventricular function by gated single-photon emission tomograph," Eur. J. Nucl. Med. 27, 1525-1530 (2000).

${ }^{2}$ B. Stefaniak, W. Cholewinski, M. Wypych, A. Olesiuk, and A. Tarkowska, "The construction of dynamic cardiac phantom for gated myocardial SPECT—A preliminary work," Eur. J. Nucl. Med. 27, 1193 (2000) (abstract).

${ }^{3}$ B. M. W. Tsui, J. A. Terry, and G. T. Gullberg, "Evaluation of cardiac cone-beam single photon emission computed tomography using observer performance experiment and receiver operating characteristic analysis," Invest. Radiol. 28, 1101-1112 (1993).

${ }^{4}$ J. Peter, D. R. Gilland, R. J. Jaszczak, and R. E. Coleman, "Fourdimensional superquadratic-based cardiac phantom for Monte Carlo simulation of radiological imaging systems," IEEE Trans. Nucl. Sci. 46, 2211-2217 (1999).

${ }^{5}$ P. H. Pretorius, M. A. King, B. M. W. Tsui, K. J. Lacroix, and W. Xia, “A mathematical model of motion of the heart for use in generating source and attenuation maps for simulating emission imaging," Med. Phys. 26, 2323-2332 (1999).

${ }^{6}$ E. J. Hoffman, P. D. Cutler, W. M. Digby, and J. C. Mazziotta, "3-D phantom to simulate cerebral blood flow and metabolic images for PET," IEEE Trans. Nucl. Sci. NS-37, 616-620 (1990).

${ }^{7}$ I. G. Zubal, C. R. Harrell, E. O. Smith, Z. Rattner, G. R. Gindi, and P. B. Hoffer, "Computerized three-dimensional segmented human anatomy," Med. Phys. 21, 299-302 (1994).

${ }^{8}$ W. P. Segars, D. S. Lalush, and B. M. W. Tsui, "A realistic spline-based dynamic heart phantom," IEEE Trans. Nucl. Sci. 46, 503-506 (1999).
${ }^{9}$ W. P. Segars, "Development of a new dynamic NURBS-based cardiactorso (NCAT) phantom," Ph.D. dissertation, The University of North Carolina, May 2001.

${ }^{10} \mathrm{~K}$. J. LaCroix and B. M. W. Tsui, "The effect of defect size, location, and contrast on the diagnosis of myocardial defects in SPECT with and without attenuation compensation," J. Nucl. Med. 37, 20 (1996).

${ }^{11}$ P. H. Pretorius, W. Xia, M. A. King, B. M. W. Tsui, T. S. Pan, and B. J. Villegas, "Determination of left and right ventricular volume and ejection fraction using a mathematical cardiac torso phantom for gated blood pool SPECT," J. Nucl. Med. 37, 97 (1996).

${ }^{12}$ D. S. Lalush and B. M. W. Tsui, "Block-iterative techniques for fast 4-D reconstruction using a priori motion models in gated cardiac SPECT," Phys. Med. Biol. 43, 875-887 (1998).

${ }^{13}$ G. R. Heyndrickx, R. W. Millard, R. J. McRitchie, P. R. Maroko, and S. F. Vatner, "Regional myocardial functional and electrophysiological alternations after brief coronary artery occlusion in conscious dogs," $\mathrm{J}$. Clin. Invest. 56, 978-985 (1975).

${ }^{14}$ E. Braunwald and R. A. Kloner, "The stunned myocardium: prolonged, postischaemic ventricular dysfunction," Circulation 66, 1146-1149 (1982).

${ }^{15}$ P. V. Ford, S. N. Chatziioannou, W. H. Moore, and R. D. Dhekne, "Overestimation of the LVEF by quantitative Gated SPECT in simulated left ventricles," J. Nucl. Med. 42, 454-459 (2001).

${ }^{16}$ K. Nakajima, J. Taki, T. Higuchi, M. Kawano, M. Taniguchi, K. Maruhashi, S. Sakazume, and N. Tonami, "Gated SPECT quantification of small hearts: mathematical simulations and clinical applications," Eur. J. Nucl. Med. 27, 1372-1379 (2000).

${ }^{17}$ G. El Fakhri, I. Buvat, P. Almeida, B. Bendriem, A. Todd-Pokropek, and H. Benali, "Should scatter be corrected in both transmission and emission data for accurate quantitation in cardiac SPET?," Eur. J. Nucl. Med. 27, 1356-1364 (2000).

${ }^{18}$ M. Potel, S. MacKay, J. Rubin, A. Aisen, and R. Sayre, “Threedimensional left ventricular wall motion in man," Invest. Radiol. 19, 499-509 (1984).

${ }^{19}$ J. Dumesnil, R. Shoucri, L. J. L. Turcot, and J. Turcot, "A mathematical model of the dynamic geometry of the intact left ventricle and its application to clinical data," Circulation 59, 1024-1034 (1979).

${ }^{20}$ M. Fogel et al., "Mechanics of the single left ventricle," Circulation 98, 330-338 (1998).

${ }^{21}$ J. Lessick, Y. Fisher, R. Beyar, S. Sideman, M. Marcus, and H. Azhari, "Regional three-dimensional geometry of the normal human left ventricle using cine computed tomography," Ann. Biomed. Eng. 24, 583594 (1996)

${ }^{22}$ J. Park, D. N. Metaxas, and L. Axel, "Quantification and visualization of the 3-D nonrigid motion of the left ventricle," Proceedings of the SPIE Medical Imaging Conference (Physiology and Function), 1997, p. 177.

${ }^{23}$ J. Park, D. N. Metaxas, A. A. Young, and L. Axel, "Deformable models with parameter functions for cardiac motion analysis from tagged MRI data," IEEE Trans. Med. Imaging 15, 278-289 (1996).

${ }^{24}$ J. Park, D. N. Metaxas, and L. Axel, "Analysis of left ventricular wall motion based on volumetric deformable models and MRI-SPAMM," Med. Image Anal. 1, 53-71 (1996).

${ }^{25}$ J. Mirro, E. W. Rogers, A. E. Weyman, and H. Feigenbaum, "Angular displacement of the papillary muscles during cardiac cycle," Circulation 60, 327-333 (1979).

${ }^{26}$ W. P. Segars, D. S. Lalush, and B. M. W. Tsui, "Modeling respiratory mechanics in the MCAT and spline-based MCAT phantom," IEEE Trans. Nucl. Sci. 1, 89-97 (2001).

${ }^{27}$ M. M. Ter-Pogossian, S. R. Bergman, and B. E. Sobel, "Influence of cardiac and respiratory motion on tomographic reconstructions of the heart: Implications for quantitative nuclear cardiology," J. Comput. Assist. Tomogr. 6, 1148-1155 (1982).

${ }^{28}$ W. H. Pres, S. A. Teukolsky, W. T. Vetterling, and B. P. Flannery, Numerical Recipes in C, 2nd ed. (Cambridge University Press, Cambridge, 1992).

${ }^{29}$ T. K. Lewellen et al., "Design of a simulation system for emission tomographs," J. Nucl. Med. 29, 871 (1988) (abstract).

${ }^{30}$ D. R. Haynor, R. L. Harrison, and T. K. Lewellen, "The use of importance sampling techniques to improve the efficiency of photon tracking in emission tomography simulations," Med. Phys. 18, 990-1001 (1991).

${ }^{31}$ M. L. Marcus, H. R. Schelbert, D. J. Skortion, and G. L. Wolf, Cardiac Imaging. A Companion to Braunwald's Heart Disease (S. Baunders Company, Philadelphia, 1991). 